\title{
Worldwide dietary and lifestyle factors associated with diabetes prevalence and total cholesterol levels: an ecological analysis
}

\author{
C. Oggioni ${ }^{1,2}$, H. Cena ${ }^{2}$, J. C. K. Wells ${ }^{3}$, K. Soroka ${ }^{1}$, J. Lara ${ }^{1}$, C. Celis Morales ${ }^{1}$ and M. Siervo ${ }^{1}$ \\ ${ }^{1}$ Human Nutrition Research Centre, Institute for Ageing and Health, Newcastle University, Campus for Ageing and \\ Vitality, Newcastle on Tyne, NE4 5PL, UK, ${ }^{2}$ Clinical Nutrition Laboratory, Department of Public Health, \\ Experimental and Forensic Medicine, University of Pavia, Pavia, IT and ${ }^{3}$ Childhood Nutrition Research Centre, \\ UCL Institute of Child Health, London, UK
}

The worldwide epidemiology of diabetes and hypercholesterolemia is changing rapidly ${ }^{(1,2)}$ as a result of the diffusion of Westernised nutritional and lifestyle patterns ${ }^{(3,4)}$. We conducted an ecological analysis to identify dietary, lifestyle and socio-economic factors associated with global distribution of diabetes prevalence and total cholesterol levels.

Country-specific estimates of diabetes prevalence and total cholesterol levels were obtained from freely available electronic databases maintained and updated by as the World Health Organisation (WHO), Food and Agriculture Organisation (FAO) and the World Bank. Data on diabetes prevalence and cholesterol concentrations were then matched to year- and country-specific food and energy availability for consumption and to year-specific information on physical inactivity, urbanisation, gross domestic product (GDP), life expectancy, and smoking. Cluster analysis was used to derive typical dietary patterns of global food consumption and their association with diabetes prevalence and total cholesterol levels was evaluated. Socio-demographic and dietary predictors of diabetes prevalence and total cholesterol levels were identified using multiple regression models.

Physical inactivity and eggs consumption emerged as predictors of diabetes and total cholesterol levels in fully-adjusted multiple regression models, respectively. Three dietary patterns (agricultural, transitional and westernised) were identified by the cluster analysis. A significant increase in diabetes prevalence and total cholesterol levels was observed as countries move from an agricultural to a westernised dietary pattern (Fig. 1).
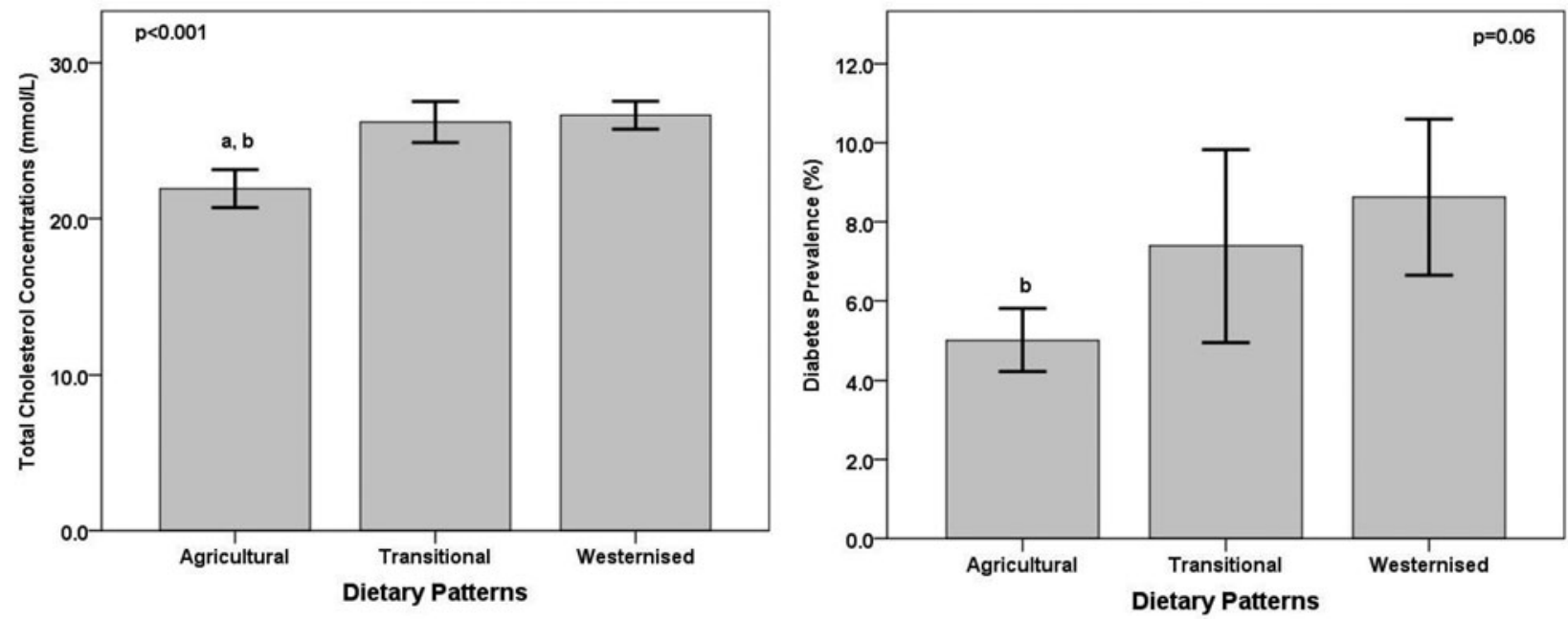

Fig. 1. Differences in total cholesterol levels and prevalence of diabetes between dietary patterns.

Prevention of physical inactivity is a global priority as closely linked to worldwide diabetes burden. The role of global consumption of eggs as a predictor of total cholesterol levels is a novel finding which requires further validation in epidemiological studies conducted in developed and developing countries.

1. Murray CJ, Lopez AD (1997) Global mortality, disability, and the contribution of risk factors: Global Burden of Disease Study. Lancet 349(9063), $1436-42$.

2. Hu FB (2011) Globalization of diabetes: the role of diet, lifestyle, and genes. Diabetes care 34(6), 1249-57.

3. Kohl HW 3rd, Craig CL, Lambert EV, Inoue S, Alkandari JR, Leetongin G, et al. (2012) The pandemic of physical inactivity: global action for public health. Lancet 380(9838), 294-305.

4. Popkin BM (1999) Urbanization, lifestyle changes and the nutrition transition. World Dev 27(11), 1905-16. 